\title{
ZERO ROTATION APROACH FOR DROOP IMPROVEMENT IN CASCADED INTEGRATORS COMBFILTER
}

\author{
Varsha Dhekekar ${ }^{1}$, Jaikaran Singh ${ }^{2}$ \\ ${ }^{I}$ Dept of Electronics and Communication Engineering, Shree Satya Sai university of technology and Medical science, \\ Sehore, MP, India \\ ${ }^{2}$ Dean faculty of Electrical \& Electronics Engineering, Shree Satya Sai university of technology and Medical \\ science, Sehore, MP, India
}

\begin{abstract}
Cascaded Integrators Comb (CIC) filter is a special class of linear phase FIR filter. It is the most simple decimation filter which does not require multipliers. Due to its simplicity, the CIC filter is usually used in the first stage of decimation. They are used in multi-rate digital signal processing systems which have different applications such as efficient filtering, sub band loading, analogue / digital conversions, audio video signals. However the filter exhibits a high pass band droop which is inacceptable in different application.
\end{abstract}

In this paper, the improvement in pass band droop is done by zero rotation approach.

Keywords-Cascaded Integrator comb filters, decimation, zero rotation

\section{INTRODUCTION}

AS given in[1], decimation filter has wide application in analog and digital system used for data rate conversion and filtering. The decimation technique using CIC filter has been discussed. Due to its limited storage, it is used in economical hardware implementation. The magnitude response of CIC filter so obtained has low attenuation in the alias band and droop in the pass band region which effects the signal obtained after decimation.

To improve this alias rejection, the no. of stages has to be increased which causes increase droop in the pass band region.

The pass band droop compensation techniques using compensators are discussed in [2], [4] \& [5]. In [2], the high order FIR compensator is designed based on maximally flat error criteria. In [5] the maximally flat compensators are designed for $2^{\text {nd }}$ order $\& 4^{\text {th }}$ order CIC filter. The weighted least -square design proposed in [4], where the compensation is combined with sharpening.

The design of Generalised Comb Filter GCF designed which quantizes the multiplier in Z- transfer function employing power of two (PO2) terms given in[3]. Using the optimization algorithm there is a improvement in pass band as well as Stop band based on efficient IIR filter proposed in [6].

The main goal of this paper is to introduce an efficient CIC filter with improvement in pass band droop. The rest of this paper is organised as follows:
The theoretical background of CIC filters in section II while Section III describes the proposed algorithm. Section IV gives the comparison of original CIC and proposed CIC filter. Discussion of the result is given in section $\mathrm{V}$.

\section{THEORITICALBACKGROUND}

The CIC filter has low pass characteristics and its structure resembles am implementation of moving average filter. It was introduced by Eugene.B. Hogenauer and is used for implementation and decimation. This filter consists of two main sections, cascaded integrator and comb separated by a down - sampler

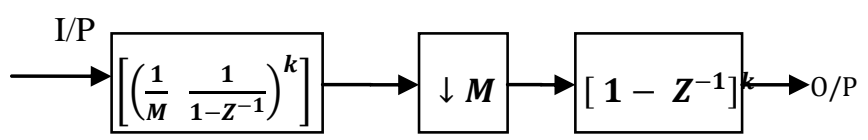

The transfer function of the resulting decimation is given by

$$
H(z)=\left(\frac{1}{M}\left[\frac{1-Z^{-M}}{1-Z^{-1}}\right]\right)^{k}
$$

Where $\mathbf{M}$ is the decimation factor $\& \mathbf{k}$ is the no. of stages respectively.

As the no. of stages are increased the attenuation in the folding bands are increased and also the pass band droop is increased which can be seen in the figure 1(a) and pass band zoom in figure 1 (b) 


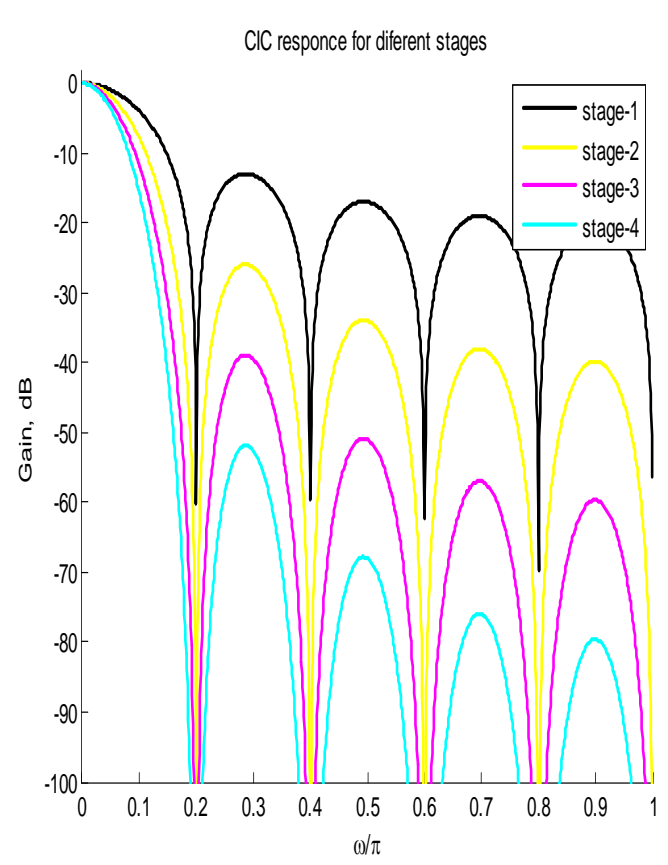

Fig. 1(a): CIC response for different stages

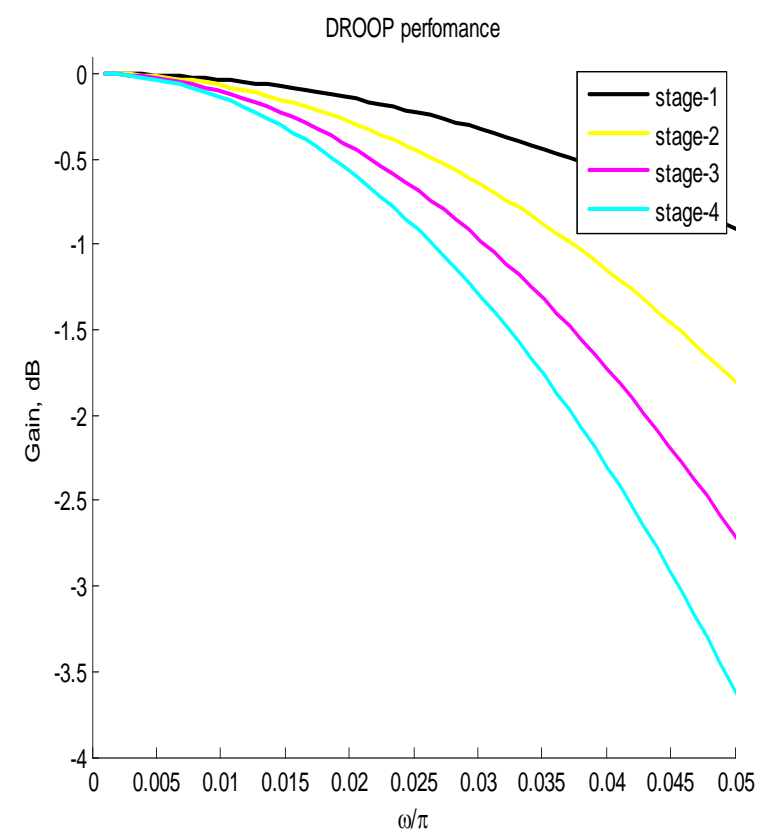

Fig. 1(b): Droop performance

Various algorithms have been proposed by different researchers. In [5] Maximally Flat CIC Compensation Filter has been proposed, in which author tries to decrease droop using narrowband and wideband compensators. In [7] proposed algorithm uses multiplier less implementation of compensator for droop improvement. Author also tries to reduce complexity. In [6] proposed algorithm uses IIR filter with CIC filter for droop improvement. It uses cascaded combination of CIC and IIR filter for droop improvement. In [8] proposed algorithm uses FIR filter with CIC filter for droop improvement. It uses cascaded combination of CIC and FIR filter for droop improvement.

\section{PROPOSED ALGORITHM}

The proposed algorithm, i.e., CIC zero rotation approach proposed rotated sinc filter which is designed such that there is a decrease in pass band droop.

By applying a clockwise rotation of $\beta$ radians to only zero of CIC filter, we obtain the following transfer function,

$$
H_{u}(z)=\frac{1}{M} \frac{1-Z^{-M} e^{j \beta M}}{1-Z^{-1} e^{j \beta}}
$$

An expression equivalent is obtained by applying opposite rotation to only zero of CIC filter we obtain the following transfer function,

$$
H_{d}(z)=\frac{1}{M} \frac{1-Z^{-M} e^{-j \beta M}}{1-Z^{-1} e^{-j \beta}}
$$

These two filters have complex co-efficient but they are cascaded, thus obtaining a filter with real co-efficient,

$$
\begin{gathered}
H_{r}(z)=H_{u}(z) H_{d}(z) \\
=\frac{1}{M^{2}} \frac{1-2 \cos (\beta M) Z^{-M}+Z^{-2 M}}{1-2 \cos (\beta) Z^{-1}+Z^{-2}}
\end{gathered}
$$

The cascade of CIC filter and filter referred by Presti as RS filter, is given as,

$$
H_{r}(z)=H_{C I C}(z) H_{r}(z)
$$

The magnitude response of the filter is given as,

$$
\mid H_{r}\left(\left.e^{j \omega}|=| \frac{1}{M^{3}} \frac{\sin (\omega M / 2)}{\sin (\omega / 2)}\right|^{k}\left|\frac{\sin \{(\omega+\beta) M / 2\}}{\sin \{(\omega+\beta) M / 2\}}\right|^{k}\left|\frac{\sin \{(\omega-\beta) M / 2\}}{\sin \{(\omega-\beta) M / 2\}}\right|^{k}\right.
$$

\section{COMPARISON OF CIC FILTER AND PROPOSED METHOD'S FILTER}

Following design parameters are being considered in simulation: the decimation factor $\mathrm{D}$ is equal to 32 , the no. of stages $\mathrm{N}$ is $5, \mathrm{~V}=4, \mathrm{f}_{\mathrm{m}}=0.02 \mathrm{q}$ factor $=0.78$ and $\alpha=0.78 *$ pi $*$ $\mathrm{f}_{\mathrm{m}}$ the pass band frequency $\omega_{\mathrm{p}}$ is $0.0078 \pi \mathrm{rad}$. 


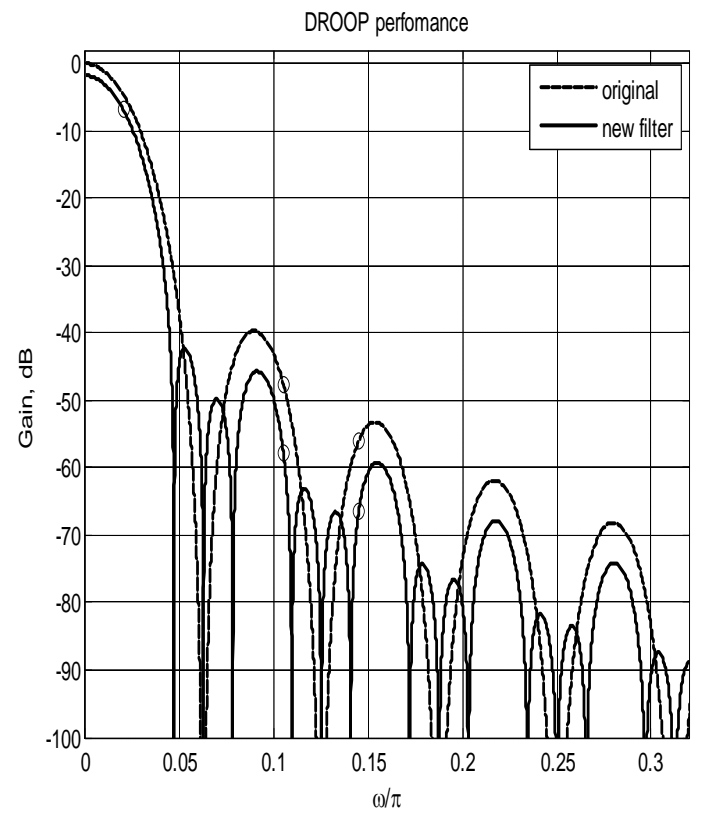

Fig. 2(a): comparison of droop performances of original \& new CIC filter

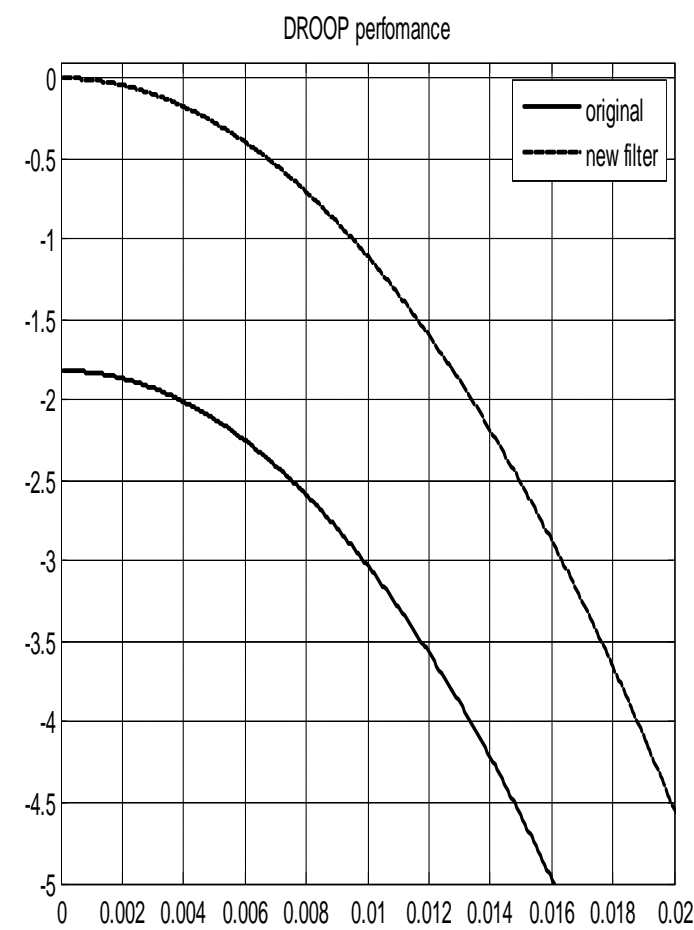

Fig. 2(b): Droop performance improvement

Fig. 2(a) illustrates the magnitude responses of both original and new CIC filters. Pass band details of original and new CIC filter is shown $n$ fig. 2 (b)

Note that at pass band frequency $\omega_{\mathrm{p}}=0.0078$ there is $1.91 \mathrm{~dB}$ improvement. For same parameters $1 \mathrm{~dB}$ improvement is shown in [5].

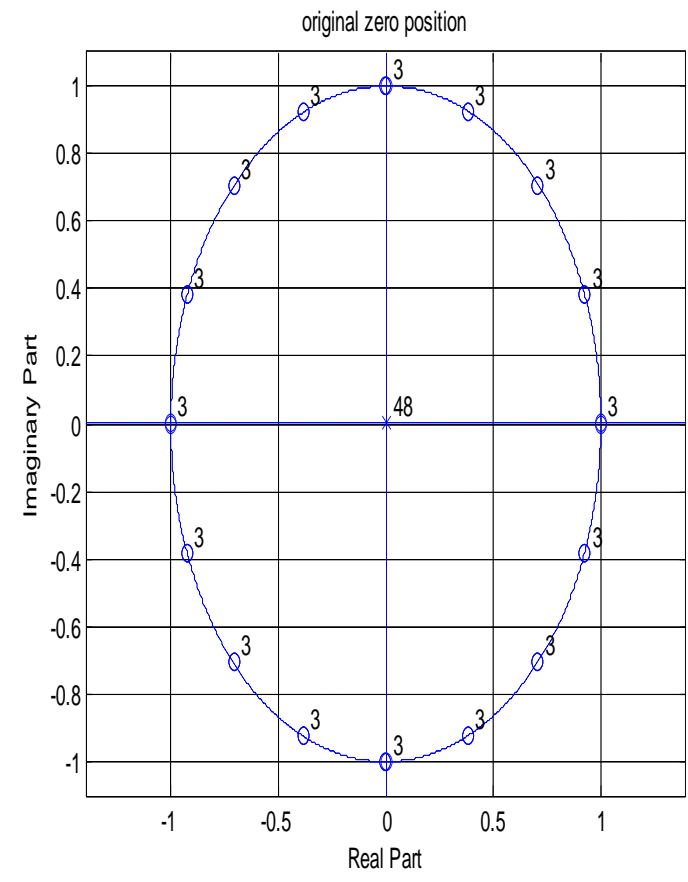

Fig. 3(a): Zeros position of original CIC filter

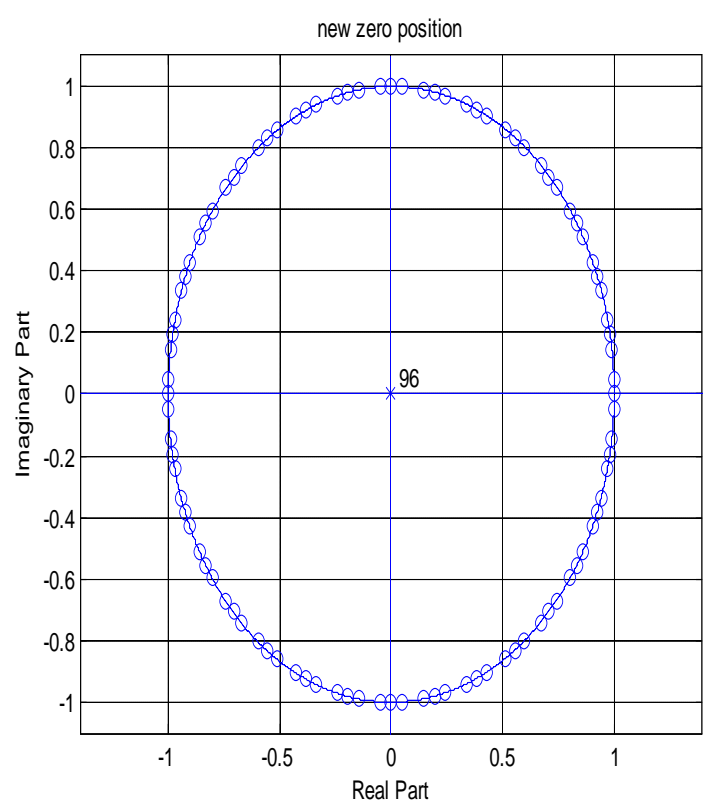

Fig. 3(b): Zeros position of new CIC filter

In Fig. 3(a) and Fig. 3(b) distribution of zeroes in existing and proposed algorithm is shown. It can be clearly seen In Fig 3(a) group of 3 zeroes are located at same position in 16 places. While in Fig 3(b) no zeroes are located at same place, which yields better distribution of zeroes that results in better response.

\section{CONCLUSIONS}

It can be clearly seen in comparison part that proposed algorithm provides better performance as compared to original filter. Better distribution of zeroes can yield better 
results. More research can be done on complexity of proposed filter.

\section{ACKNOWLEDGMENTS}

The authors would like to thank the anonymous reviewers and the Associate Editor for their helpful comments and recommendations, which helped to improve the presentation of this brief.

\section{REFERENCES}

[1]. Vishal Awasthi\& Trishla Devi Gupta "Analysis ofCascaded Integrator Comb (CIC)Decimation Filter in Efficient Compensation" International Journal of Electronics Engineering, 3 (2), 2011, pp. 203-208.

[2]. Goran Molnar, Member, IEEE, and Mladen Vucic, Member, IEEE "Closed-Form Design of CIC Compensators Based

on Maximally Flat Error Criterion" IEEE TRANSACTIONS ON CIRCUITS AND SYSTEMS - II: express briefs, vol. 58, no. 12, December 2011.

[3]. G. Jovanovic Dolecek, Senior Member, IEEE, and Massimiliano Laddomada, Senior Member, IEEE "An Economical Class of Droop-CompensatedGeneralized Comb Filters: Analysis and Design" IEEE TRANSACTIONS ON CIRCUITS AND SYSTEMS-II: EXPRESS BRIEFS, VOL. 57, NO. 4, APRIL 2010

[4]. Goran Molnar, Member, IEEE, Matija Glavinic Pecotic and Mladen Vucic, Member, IEEE " Weighted Least Squares Design of Sharpened CIC filter, MIPRO 2013, May 20-24, 2013, Opatija, Croatia.

[5]. Alfonso Fernandez-Vazquez, Member, IEEE, and Gordana Jovanovic Dolecek, Senior Member, IEEE"Maximally Flat CIC Compensation Filter: Designand Multiplierless Implementation" IEEE TRANSACTIONS ON CIRCUITS AND SYSTEMS-II: EXPRESS BRIEFS, VOL. 59, NO. 2, FEBRUARY 2012

[6]. Alfonso Fernandez-VazquezGordana Jovanovic Dolecek, "Passband and Stopband CIC Improvement Based on Efficient IIR Filter Structure" 978-1-4244-7773-9/10/ @2010 IEEE

[7]. G. Jovanovic Dolecek, S.K. Mitra, "Two-stage CICbased decimator with improved characteristics", IET Signal Process., 2010, Vol. 4, Iss. 1, pp. 22-29

[8]. Vishal Awasthi1 \& Trishla Devi Gupta, "Analysis of Cascaded Integrator Comb (CIC) Decimation Filter in Efficient Compensation", International Journal of Electronics Engineering, 3 (2), 2011, pp. 203-208 